\title{
A Plasma Metabolomic Investigation of Colorectal Cancer Patients by Liquid Chromatography-Mass Spectrometry
}

\author{
Yousuke Fukui ${ }^{*}$ and Kohji Itoh
}

\author{
Department of Medicinal Biotechnology, Institute for Medicinal Resources, Graduate School of Pharmaceutical \\ Sciences, The University of Tokushima, 1-78 Sho-machi, Tokushima 770-8505, Japan
}

\begin{abstract}
Due to hypoxic conditions, colorectal cancer (CRC) tissues have a specific metabolism compared with normal mucosa, and this study aimed to investigate blood plasma metabolite differences between CRC patients and healthy subjects. Non-targeted methods have previously been used to characterize CRC disease diagnosis and identify small molecules in biological samples, but here we used a metabolomic approach based on liquid chromatography-mass spectrometry (LC-MS) of blood plasma from CRC patients $(\mathrm{n}=10)$ and healthy subjects $(\mathrm{n}=10)$. The analysis detected 130 identified compounds including free amino acids, fatty acids and nucleotides. Of these, many free amino acids were decreased in CRC patients while nucleotide-related and stress-related metabolites were increased in CRC patients compared with healthy subjects. Multivariate analysis clearly separated CRC patients and healthy subjects. These results demonstrate that plasma metabolite would reflects tumoral specific metabolism and metabolomic profiling of blood plasma using LC-MS is a valuable tool for characterizing CRC patients based on plasma metabolite diagnostic markers.
\end{abstract}

Keywords: Blood plasma, colorectal cancer, metabolome.

\section{INTRODUCTION}

Colorectal cancer (CRC) is a major cause of worldwide mortality and morbidity, and is the third most common cancer and the second leading cause of cancer death $[1,2]$. Despite recent advances in management and medical examination, CRC mortality levels are not decreasing. Presymptomatic screening aims to detect early stage CRC or its precursor lesions for improved cancer-specific survival and reduced treatment-related morbidity. Currently, fecal occult blood tests are widely used for CRC screening, while colonoscopy, flexible sigmoidscopy and double contrast barium enema are employed for close examination. Genomic and proteomic techniques have identified many CRC serum, tissue and fecal-based biomarkers including carcinoembryonic antigen (CEA) and carbohydrate antigen 19-9 (CA19-9); despite their limitations, only serum CEA and fecal-based genetic markers are clinically useful $[3,4]$. CEA is easy to detect in blood samples, but it suffers from low specificity and is therefore not useful as a screening or prognostic test.

It is generally believed that the comprehensive detection and characterization of endogenous metabolites that differ between CRC patients and healthy subjects may be used for monitoring the states, prognosis and therapy of cancer patients. Recently, metabolite profiling between tumoral tissue and normal mucosa from CRC patients found significant variations [5-8]. These reports suggest that cancerous tissues up-regulate both the energy and amino acid metabolisms. It will be possible to diagnose cancer patients using these cancer specific metabolites, for example amino

*Address correspondence to this author at the Department of Medicinal Biotechnology, Institute for Medicinal Resources, Graduate School of Pharmaceutical Sciences, The University of Tokushima, 1-78 Sho-machi, Tokushima 770-8505, Japan; Tel: +81 886337290; Fax: +81 886337290; Email: y-fukui@taiho.co.jp acids and other metabolite, from cancerous tissue samples. However, the analysis of cancerous tissues requires invasive tissue sampling from CRC patients.

Human blood contains a wide variety of chemically diverse low molecular weight compounds (LMCs), the metabolites, which can be detected and analyzed in different ways [9-12]. Studies of metabolic differences between CRC patients and healthy subjects are a growing area of research $[5,6,13,14]$, yet the differences have rarely been revealed in plasma metabolomic investigations $[10,15]$. In this study, we hypothesized that the global analysis of metabolites in blood plasma would define metabolic signatures that discriminate CRC patients from healthy subjects and plasma metabolites correlate with CRC clinicopathological characteristics. To confirm this and compare identified metabolites with reported tumoral tissue metabolite profiles of CRC and normal mucosa, we conducted the metabolomic analysis of blood plasma LMCs from CRC patients and healthy subjects using liquid chromatography-time of flight/mass spectrometry (LC-TOF/MS).

Metabolomics, a relatively new technology, is the biological consequence of genomic and environmental interaction. It is defined as the non-targeted quantitative analysis of tissue and bio-fluids for endogenous LMCs. The assessment of endogenous LMCs and their intermediates is important for understanding biological regulation and control in living organisms. Using metabolomic approaches, it is possible to detect many related endogenous metabolites, distinguish disease states, detect pharmacological and/or toxicological effects, and to conduct research on biomarkers [16]. The potential of metabolomics has recently been demonstrated in biomedical sciences and toxicity studies [12, 17-20]. In previous studies, metabolomic data were generated by nuclear magnetic resonance $[6,11,13]$, gas chromatography-mass spectrometry $[9,10,15]$, capillary 
electrophoresis-mass spectrometry [7, 12], and liquid chromatography-mass spectrometry (LC-MS) $[9,16,20]$. When these platforms were compared, LC-MS was recommended for the analysis of LMCs containing intermediates [21]. For the current study, we chose the LC-TOF/MS method for reasons of sensitivity, repeatability of retention time, and optimization of data acquisition.

This report utilized LC-TOF/MS to compare plasma metabolite levels between CRC patients and healthy subjects. Metabolomic data were analyzed by statistical methods to characterize plasma metabolomic features of CRC patients. We aimed to demonstrate the use of LCTOF/MS for CRC plasma metabolic analysis and to provide a diagnostic model and explore the potential metabolic biomarkers distinguishing CRC patients from healthy subjects.

\section{MATERIALS AND METHODS}

\subsection{Materials}

The reference compounds were purchased from Wako Pure Chemical (Osaka, Japan), Sigma (St. Louis, MO), MP biomedicals (Solon, OH), and Bachem (Torrance, CA). The compounds and reagents were all of analytical grade. Stock solutions of reference compounds were prepared either in Milli-Q water or methanol at a concentration of $1 \mathrm{mg} / \mathrm{mL}$. High performance liquid chromatography (HPLC) grade acetonitrile and methanol were from Merck (Darmstadt, Germany). All other analytical grade chemicals were from Wako Pure Chemical.

Fresh frozen EDTA anti-coagulated blood plasmas were purchased from C-C Biotech (Valley Center, CA). Blood plasmas from $10 \mathrm{CRC}$ patients and 10 healthy subjects were used for non-targeted analysis. Blood plasmas were stored at $-80^{\circ} \mathrm{C}$ until analysis. They were then thawed at room temperature, centrifuged at $1900 \times g$ for $15 \mathrm{~min}$, and the supernatants used for analysis. The backgrounds of analyzed CRC patients and healthy subjects are summarized in Table $\mathbf{1}$.

A $100 \mu \mathrm{L}$ aliquot of blood plasma were deproteonized by $100 \mu \mathrm{L} \mathrm{CH}_{3} \mathrm{CN}$. After the addition of $\mathrm{CH}_{3} \mathrm{CN}$, the mixture

Table 1. Summary of Characteristics of CRC Patients and Healthy Subjects

\begin{tabular}{|c|c|c|}
\hline Clinical Features & Number & \\
\hline $\begin{array}{l}\text { Mean age (range) } \\
\text { Gender (male/female) } \\
\text { BMI } \\
\text { TNM Stage } \\
\text { T } 3 \\
4 \\
\text { N } 1 \\
2 \\
\text { M } 0 \\
\text { X }\end{array}$ & $\begin{array}{l}62.8 \pm 11.7 \\
10 / 0 \\
24.78 \pm 2.30 \\
\\
8 \\
2 \\
8 \\
2 \\
7 \\
3\end{array}$ & $(44-76)$ \\
\hline $\begin{array}{l}\text { Healthy Subjects } \\
\text { Mean age (range) } \\
\text { Gender (male/female) } \\
\text { BMI }\end{array}$ & $\begin{array}{l}51.0 \pm 6.1 \\
10 / 0 \\
24.37 \pm 2.70\end{array}$ & $(42-61)$ \\
\hline
\end{tabular}

was vortexed for $30 \mathrm{~s}$ and centrifuged at $1900 \times g$ for $5 \mathrm{~min}$. The separated upper layer was then injected into the LCTOF/MS.

\subsection{LC-TOF/MS Analysis}

A $5 \mu \mathrm{L}$ aliquot of deproteonized sample was injected by an Agilent 1200 series autosampler (Agilent technologies, Santa Clara, CA) into an Agilent 1200 series HPLC equipped with a ODS-100V column $(2.0 \mathrm{~mm} \times 150 \mathrm{~mm}, 3$ $\mu \mathrm{m}$; Toso, Tokyo, Japan). The chromatographic separations were achieved at a column temperature of $35^{\circ} \mathrm{C}$ and flow rate of $0.2 \mathrm{~mL} / \mathrm{min}$. Mobile phase A was water with $0.1 \%$ formic acid and mobile phase B was methanol with $0.1 \%$ formic acid. A three-step gradient was formed from a $0 \%$ mobile phase B held for $10 \mathrm{~min}$, a $0 \%$ to $100 \%$ mobile phase $\mathrm{B}$ over $15 \mathrm{~min}$, and the final composition held for $10 \mathrm{~min}$ before returning to the initial conditions. The initial condition was held for $15 \mathrm{~min}$ before the next injection. The column efflux was introduced into the ion source of a LCTOF/MS on Q-STAR Elite (Applied Biosystems, Foster City, CA). Positive electrospray ionization MS data were acquired from separate injections from $\mathrm{m} / \mathrm{z} 50$ to 1000 at a 1 $\mathrm{s}$ accumulation. Instrumental settings were as follows: capillary temperature: $450^{\circ} \mathrm{C}$, ionspray voltage: $5500 \mathrm{~V}$. The mass calibration was carried out using calibrator CsI $(\mathrm{m} / z=$ 132.9049) and iPD1 $(\mathrm{m} / \mathrm{z}=829.5393)$, injected by an infusion pump before each sample injection. All blood plasma samples were injected in triplicate, to allow identification of the noise peaks.

\subsection{Data Processing and Multivariate Modeling}

Collected data were used for analysis after mass recalibration using the calibrator spectrum. Data obtained from HPLC-TOF/MS were aligned using Marker View software (Applied Biosystems) as follows: mass tolerance, $10 \mathrm{ppm}$; noise threshold, 5; minimum peak width, 5ppm; maximum peak number, 20,000. After alignment, the intensity of each peak was normalized by the total intensity of injection. The normalized intensity of each sample was represented by JMP, version 7.0.1 (SAS institute, Cary, NC) and statistical analyses were performed using JMP. The peak responses of triplicate analysis were estimated; those higher than a constant validity $>20$ were deleted as noise peaks. Isotope peaks were also deleted by the retention time and spectra patterns. The selected peaks were assigned by a local data library, prepared using standard sample analysis and spectra from the data-base. To compare metabolite profiles of CRC patients and healthy subjects, statistical testing including t-tests, unsupervised principal component analysis (PCA), and hierarchical clustering analysis was conducted.

\section{RESULTS}

Metabolic profiles of blood plasma samples were analyzed using LC-TOF/MS. Detected peaks were aligned using Marker View software, and noise peaks and isotope peaks were estimated and deleted. After the selection of 4,190 peaks from the 20,000 detected peaks and 130 of these were identified using spectra and retention time recorded in a local library, and open data-bases. The 130 peaks are summarized in Table 2 , but 551 unidentified significant $(\mathrm{P}<1.22 \mathrm{E}-5)$ peaks were not identified. Typical selective ion chromatograms of identified amino acids from a gradient 
elution of $0.1 \%$ aqueous formic acid and acetonitrile as the mobile phase are shown in Fig. (1). Although, many amino acids were eluted at an early retention time, because of their hydrophilic physicality, it could identified by their own $m / z$. The linearity of this LC-TOF/MS method was estimated by a standard addition to a pooled control plasma sample. All correlation factors exceeded 0.99 , and variations in retention time over three replicated runs were within $0.1 \mathrm{~min}$.

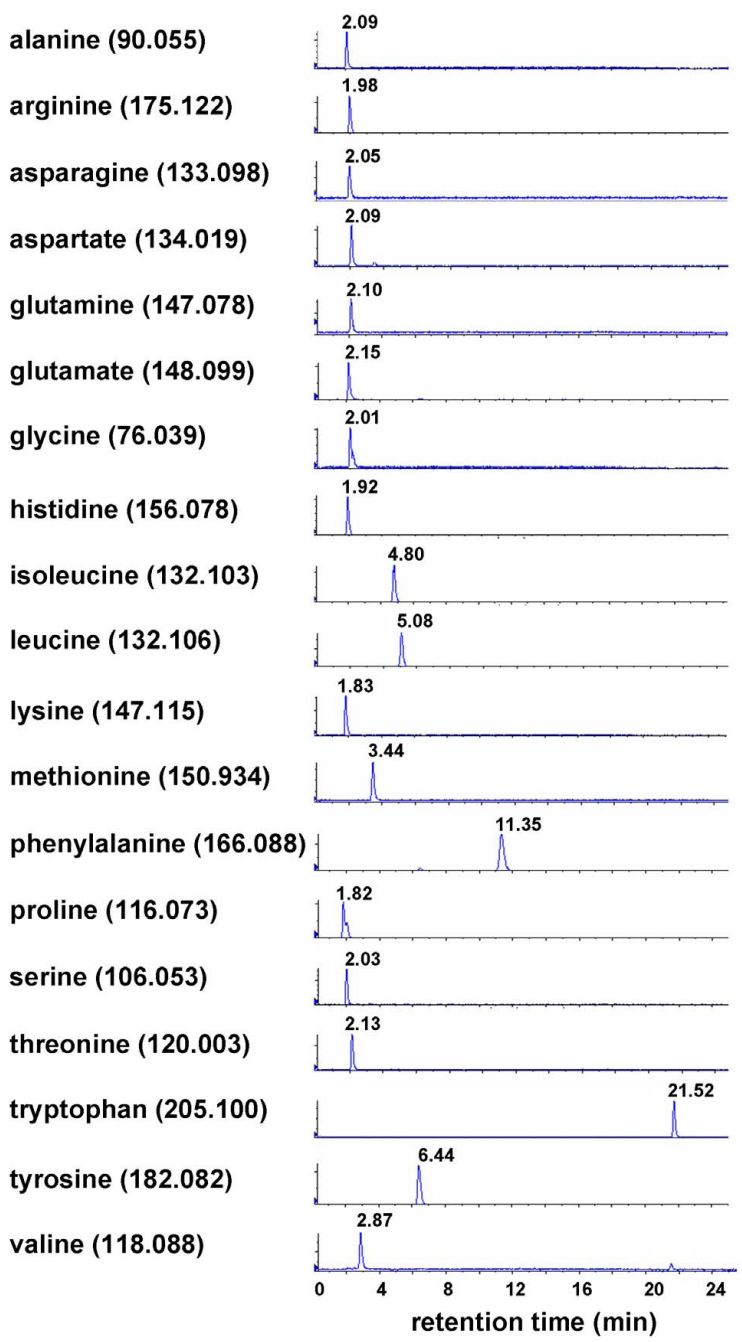

Fig. (1). Selective ion chromatograms for standard solution of low molecular weight compounds (LMCs) in positive electro spray ionization mode.

Selected peaks of differential LMCs between the CRC patients group and healthy subjects group were used to construct a specific peak describing the biochemical composition of each plasma sample. The responses of the peaks were normalized to the total sum of each sample to compensate for differences in injection response. After normalization, the t-test was performed to compare differences between the two groups (Table 2). Decreases in many free amino acids levels were observed in CRC patients, while many free fatty acid levels were significantly increased in the CRC group compared with healthy subjects. Three free amino acids, arginine, histidine, and threonine, were strongly and significantly decreased, with 10 free amino acids including aspartate, methionine, and glycine being moderately or weakly but still significantly decreased $(\mathrm{p}<0.05)$ in CRC patients compared with healthy subjects. An accumulation of several amino acid metabolites such as p-cresol sulfate, kinurenine, phenylacetylglutamine, 2aminobutylate, and $\mathrm{N}$-acethyl alanine were observed in CRC patients $(\mathrm{p}<0.05)$. Twelve free fatty acids including eicosapentaenoate, 3-carboxy-4-methyl-5-propyl-2-furanpropanoate, isovalerate, eicosenoate, docosahexaenoate, linolate, undecanoate, dihomo-linolenate, docosapentaenoate, 10-hepatadecanoate, 5-dodecenoate and 10-undecenoate were moderately or weakly but significantly increased in the CRC patients group $(\mathrm{p}<0.05)$. Laurylcarnitine, 1-oleoylglycerophosphocholine, 1-myristoylglycerophosphocholine, 1-stearoylglycerophosphocholine, hyodeoxycholate, cholate, and androsterone sulfate were significantly decreased. Several peptides derived from fibrinogen, ADpSGEGDFXA EGGGVR and DSGEGDFXAEGGGVR, were elevated in the CRC patients group as well as several nucleotides/ nucleosides including 1-methyladenosine and adenosine 5'monophosphate (weak significance except 1-methyl adenosine). 132 and 308 unidentified peaks were significantly increased and decreased, respectively. The expressed tendency and significance of eight LMCs, four identified amino acids and four unidentified LMCs, $m / z=224.868$, 96.922, 416.809, and 934.221, between CRC patients and healthy subjects are shown in Fig. (2). Plasma levels of several LMCs were clearly distinct between CRC patients and healthy subjects. Interestingly, no LMC peaks of $\mathrm{m} / \mathrm{z}=$ 943.221 were detected in plasma from CRC patients.

To characterize CRC patients from healthy subjects using metabolomic analysis of blood plasma samples, unsupervised PCA and hierarchical clustering were carried out. The scores plot based on all detected peaks from plasma is shown in Fig. (3) and reveals a complete separation between the two groups. Hierarchical clustering of identified blood plasma metabolites also resulted in a perfect separation of CRC patients and healthy subjects (Fig. 4). Most free amino acids were clustered in the lower half of the figure, and most free fatty acids were clustered in the upper half.

\section{DICUSSION}

Our current work demonstrates that large scale blood plasma metabolic profiling using LC-TOF/MS and library annotation yields numerous significant differences between CRC patients and healthy subjects. The concentrations of some amino acids (arginine, histidine, and threonine), lipids (androsterone sulfate, glycerolipid metabolites, and 1oleoylglycerophosphocholine) were significantly decreased, while p-cresol sulfate, 3-carboxy-4-methyl-5-propyl-2furanpropanoate, cortisol, 1-methyladenosine were significantly increased in blood plasma samples of CRC patients compared with healthy subjects.

Recently, several studies undertook metabolic profiling of CRC tumor tissues and normal mucosa. Hirayama et al. reported a significant change in energy metabolism because 
Table 2. Endogenous Plasma Metabolites Responses of the CRC Patients and Healthy Subjects

\begin{tabular}{|c|c|c|c|c|c|c|c|c|c|c|}
\hline \multirow{2}{*}{ Metabolite } & \multirow{2}{*}{$m / z$} & \multicolumn{3}{|c|}{ CRC Group } & \multicolumn{3}{|c|}{ HS Group } & \multirow{2}{*}{$\frac{\text { Fold Change }}{\text { CRC/HS }}$} & \multirow{2}{*}{ p Value } & \\
\hline & & Mean & \pm & SD & Mean & \pm & SD & & & \\
\hline \multicolumn{11}{|l|}{ Amino Acid Metabolism } \\
\hline glycine & 76.039 & 1.59 & \pm & 0.37 & 2.83 & \pm & 0.73 & 0.56 & $1.49 \mathrm{E}-04$ & $* *$ \\
\hline serine & 106.053 & 0.87 & \pm & 0.46 & 1.01 & \pm & 0.30 & 0.86 & 0.4342 & \\
\hline threonine & 120.003 & 3.20 & \pm & 1.16 & 8.19 & & 2.01 & 0.39 & $2.28 \mathrm{E}-06$ & $* * *$ \\
\hline betaine & 118.088 & 207.63 & \pm & 93.98 & 333.81 & \pm & 153.93 & 0.62 & 0.0401 & $*$ \\
\hline alanine & 90.055 & 17.99 & \pm & 10.63 & 27.69 & \pm & 8.57 & 0.65 & 0.0375 & $*$ \\
\hline asparatic acid & 134.019 & 4.56 & \pm & 2.64 & 11.97 & \pm & 3.58 & 0.38 & $5.24 \mathrm{E}-05$ & $* *$ \\
\hline asparagine & 133.098 & 0.53 & \pm & 0.45 & 1.52 & \pm & 0.68 & 0.35 & 0.0013 & $*$ \\
\hline beta-alanine & 91.054 & 18.75 & \pm & 1.51 & 18.41 & \pm & 1.21 & 1.02 & 0.5804 & \\
\hline glutamic acid & 148.099 & 5.99 & \pm & 2.47 & 8.35 & \pm & 2.75 & 0.72 & 0.0582 & \\
\hline glutamine & 147.078 & 134.77 & \pm & 39.32 & 182.14 & \pm & 32.06 & 0.74 & 0.0085 & * \\
\hline pyroglutamine & 129.065 & 40.99 & \pm & 26.31 & 60.72 & \pm & 30.60 & 0.68 & 0.1397 & \\
\hline histidine & 156.078 & 20.76 & \pm & 3.73 & 43.80 & \pm & 9.85 & 0.47 & $1.82 \mathrm{E}-06$ & $* * *$ \\
\hline urocanate & 139.114 & 1.75 & \pm & 0.38 & 1.88 & \pm & 0.16 & 0.93 & 0.3159 & \\
\hline lysine & 147.115 & 13.36 & \pm & 2.34 & 13.21 & \pm & 3.18 & 1.01 & 0.9105 & \\
\hline pipecolate & 130.051 & 174.65 & \pm & 48.47 & 265.51 & \pm & 38.51 & 0.66 & 2.03E-04 & ** \\
\hline phenylalanine & 166.088 & 4.94 & \pm & 7.44 & 6.12 & \pm & 9.98 & 0.81 & 0.7686 & \\
\hline tyrosine & 182.082 & 13.47 & \pm & 17.49 & 18.88 & \pm & 18.38 & 0.71 & 0.5088 & \\
\hline p-cresol sulfate & 189.161 & 18.73 & \pm & 4.78 & 0.00 & \pm & 0.00 & --- & $3.01 \mathrm{E}-10$ & $* * *$ \\
\hline phenylacetate & 137.136 & 3.52 & \pm & 0.48 & 3.60 & \pm & 0.35 & 0.98 & 0.6811 & \\
\hline phenylacetylglutamine & 265.204 & 9.76 & \pm & 0.92 & 7.85 & \pm & 1.21 & 1.24 & 8.99E-04 & ** \\
\hline 3-indoxyl sulfate & 214.079 & 0.01 & \pm & 0.03 & 0.43 & \pm & 0.49 & 0.03 & 0.0134 & $*$ \\
\hline indolepropionate & 190.079 & 2.18 & \pm & 1.76 & 5.36 & \pm & 2.47 & 0.41 & 0.0039 & $*$ \\
\hline kynurenine & 209.057 & 2.00 & \pm & 1.37 & 0.25 & \pm & 0.17 & 8.07 & $7.80 \mathrm{E}-04$ & $* *$ \\
\hline tryptophan & 205.100 & 221.08 & \pm & 192.69 & 547.23 & \pm & 268.76 & 0.40 & 0.0059 & $*$ \\
\hline valine & 118.088 & 277.10 & \pm & 137.54 & 447.37 & \pm & 189.08 & 0.62 & 0.0334 & $*$ \\
\hline leucine & 132.106 & 142.54 & \pm & 317.08 & 174.08 & \pm & 158.64 & 0.82 & 0.7817 & \\
\hline isoleucine & 132.103 & 4.71 & \pm & 8.05 & 14.19 & \pm & 18.21 & 0.33 & 0.1495 & \\
\hline 3-methyl-2-oxobutyrate & 117.092 & 6.17 & \pm & 1.26 & 5.64 & \pm & 1.04 & 1.09 & 0.3239 & \\
\hline beta-hydroxyisovalerate & 119.086 & 0.24 & \pm & 0.25 & 0.11 & \pm & 0.15 & 2.27 & 0.1597 & \\
\hline $\mathrm{N}$-acetylalanine & 132.104 & 13.47 & \pm & 14.50 & 0.42 & \pm & 0.97 & 31.72 & 0.0109 & * \\
\hline methionine & 150.934 & 2.47 & \pm & 0.28 & 3.07 & \pm & 0.26 & 0.80 & $8.30 \mathrm{E}-05$ & $* *$ \\
\hline urea & 60.902 & 1.08 & \pm & 0.87 & 0.71 & \pm & 0.52 & 1.53 & 0.2624 & \\
\hline arginine & 175.122 & 125.49 & \pm & 31.95 & 339.59 & \pm & 62.32 & 0.37 & $1.50 \mathrm{E}-08$ & $* * *$ \\
\hline dimethylarginine & 203.153 & 19.47 & \pm & 5.60 & 15.57 & \pm & 1.49 & 1.25 & 0.0473 & $*$ \\
\hline proline & 116.073 & 2.68 & \pm & 1.16 & 4.82 & \pm & 1.30 & 0.56 & 0.0011 & $*$ \\
\hline homostachydrine* & 158.118 & 3.81 & \pm & 6.30 & 2.86 & \pm & 2.92 & 1.33 & 0.6701 & \\
\hline stachydrine & 144.102 & 34.86 & \pm & 29.43 & 30.22 & \pm & 33.36 & 1.15 & 0.7449 & \\
\hline trans-4-hydroxyproline & 132.066 & 7.08 & \pm & 3.97 & 10.42 & \pm & 8.68 & 0.68 & 0.2826 & \\
\hline creatine & 132.077 & 235.86 & \pm & 155.81 & 259.99 & \pm & 66.14 & 0.91 & 0.6575 & \\
\hline creatinine & 114.067 & 180.74 & \pm & 48.46 & 200.80 & \pm & 42.17 & 0.90 & 0.3366 & \\
\hline 2-aminobutyrate & 104.108 & 40.05 & \pm & 8.98 & 46.78 & \pm & 13.77 & 0.86 & 0.0181 & * \\
\hline \multicolumn{11}{|l|}{ Peptide } \\
\hline glutamylvaline & 247.150 & 2.69 & \pm & 0.23 & 3.14 & \pm & 0.37 & 0.85 & 0.0041 & $*$ \\
\hline pro-hydroxy-pro & 229.154 & 34.98 & \pm & 31.07 & 14.42 & \pm & 9.95 & 2.42 & 0.0617 & \\
\hline gamma-glutamylglutamine & 276.196 & 70.03 & & 59.90 & 0.00 & & 0.00 & --- & 0.0016 & * \\
\hline gamma-glutamylleucine & 261.135 & 25.10 & \pm & 4.51 & 34.23 & \pm & 5.68 & 0.73 & $8.80 \mathrm{E}-04$ & * \\
\hline gamma-glutamylphenylalanine & 295.132 & 1.03 & \pm & 0.88 & 2.07 & \pm & 1.23 & 0.49 & 0.0422 & $*$ \\
\hline gamma-glutamyltyrosine & 311.211 & 99.64 & \pm & 6.01 & 95.76 & \pm & 6.86 & 1.04 & 0.1948 & \\
\hline ADpSGEGDFXAEGGGVR & 809.591 & 6.84 & \pm & 4.57 & 2.54 & \pm & 2.63 & 2.69 & 0.0189 & $*$ \\
\hline ADSGEGDFXAEGGGVR & 733.345 & 5.74 & \pm & 12.68 & 1.14 & \pm & 2.51 & 5.02 & 0.2758 & \\
\hline DSGEGDFXAEGGGVR & 769.412 & 930.50 & \pm & 572.30 & 103.00 & \pm & 25.56 & 9.03 & $2.39 \mathrm{E}-04$ & $* *$ \\
\hline \multicolumn{11}{|l|}{ Calbohydrate Metabolism } \\
\hline fructose & 181.086 & 28.03 & \pm & 27.99 & 0.35 & \pm & 0.23 & 81.21 & 0.0058 & * \\
\hline glucose & 181.027 & 0.21 & \pm & 0.18 & 0.12 & \pm & 0.15 & 1.76 & 0.2278 & \\
\hline 1,6 -anhydroglucose & 163.124 & 0.00 & \pm & 0.00 & 0.34 & \pm & 0.50 & 0.00 & 0.0480 & $*$ \\
\hline mannose & 181.027 & 0.21 & \pm & 0.18 & 0.12 & \pm & 0.15 & 1.76 & 0.2278 & \\
\hline pyruvate & 89.061 & 30.21 & \pm & 2.44 & 30.83 & \pm & 2.17 & 0.98 & 0.5509 & \\
\hline glycerate & 107.029 & 6.45 & \pm & 0.58 & 5.99 & \pm & 0.40 & 1.08 & 0.0500 & * \\
\hline \multicolumn{11}{|l|}{ Lipid Metabolism } \\
\hline isovalerate & 103.074 & 1.74 & \pm & 0.76 & 0.42 & \pm & 0.71 & 4.15 & $7.98 \mathrm{E}-04$ & ** \\
\hline caprate & 173.071 & 2.49 & \pm & 3.40 & 0.00 & \pm & 0.00 & --- & 0.0324 & $*$ \\
\hline laurate & 201.180 & 0.80 & \pm & 0.36 & 0.71 & \pm & 0.31 & 1.12 & 0.5758 & \\
\hline palmitate & 257.233 & 0.99 & \pm & 1.70 & 0.79 & \pm & 0.82 & 1.24 & 0.7521 & \\
\hline
\end{tabular}


(Table 2) continued....

\begin{tabular}{|c|c|c|c|c|c|c|c|c|c|c|}
\hline \multirow{2}{*}{ Metabolite } & \multirow{2}{*}{$m / z$} & \multicolumn{3}{|c|}{ CRC Group } & \multicolumn{3}{|c|}{ HS Group } & \multirow{2}{*}{$\begin{array}{c}\text { Fold Change } \\
\text { CRC/HS }\end{array}$} & \multirow{2}{*}{\multicolumn{2}{|c|}{ p Value }} \\
\hline & & Mean & \pm & SD & Mean & \pm & SD & & & \\
\hline Lipid Metabolism & & & & & & & & & & \\
\hline stearate & 285.142 & 1.85 & \pm & 1.30 & 1.85 & \pm & 1.76 & 1.00 & 0.9995 & \\
\hline eicosenoate & 311.168 & 32.14 & \pm & 11.01 & 12.43 & \pm & 11.02 & 2.59 & $8.37 \mathrm{E}-04$ & $* *$ \\
\hline 10-heptadecenoate & 269.129 & 0.39 & \pm & 0.59 & 0.00 & \pm & 0.00 & -- & 0.0519 & \\
\hline 5-dodecenoate & 199.170 & 5.04 & \pm & 0.45 & 4.65 & \pm & 0.42 & 1.08 & 0.0564 & \\
\hline 10-undecanoate & 185.129 & 10.69 & \pm & 10.14 & 4.13 & \pm & 2.00 & 2.59 & 0.0597 & \\
\hline oreate & 283.263 & 108.78 & \pm & 57.79 & 54.20 & \pm & 77.54 & 2.01 & 0.0911 & \\
\hline myristleate & 227.239 & 1.74 & \pm & 0.56 & 1.35 & \pm & 0.77 & 1.29 & 0.2089 & \\
\hline palmitoleate & 255.233 & 13.67 & \pm & 7.75 & 8.79 & \pm & 16.20 & 1.55 & 0.4015 & \\
\hline eicosapentaenoate & 303.227 & 304.91 & \pm & 133.04 & 90.47 & \pm & 102.61 & 3.37 & $7.75 \mathrm{E}-04$ & $* *$ \\
\hline docosahexaenoate & 329.249 & 37.99 & \pm & 27.98 & 6.86 & \pm & 11.12 & 5.54 & 0.0043 & $*$ \\
\hline linoleate & 281.248 & 88.46 & \pm & 48.49 & 26.82 & \pm & 42.81 & 3.30 & 0.0075 & $*$ \\
\hline dihomo-linolenate & 309.147 & 23.04 & \pm & 11.76 & 9.29 & \pm & 14.10 & 2.48 & 0.0293 & $*$ \\
\hline docosapentaenoate & 331.203 & 1.24 & \pm & 0.46 & 0.74 & \pm & 0.47 & 1.67 & 0.0295 & $*$ \\
\hline linolenate & 279.165 & 6.82 & \pm & 3.72 & 9.66 & \pm & 3.20 & 0.71 & 0.0830 & \\
\hline dihomo-alpha-linolenate & 307.676 & 45.16 & \pm & 3.95 & 42.58 & \pm & 3.89 & 1.06 & 0.1576 & \\
\hline undecenoate & 187.087 & 67.75 & \pm & 74.28 & 0.02 & \pm & 0.05 & 4336.81 & 0.0099 & $*$ \\
\hline nonadecanoate & 299.657 & 17.49 & \pm & 2.14 & 16.36 & \pm & 1.75 & 1.07 & 0.2136 & \\
\hline margarate & 271.268 & 3.23 & \pm & 0.77 & 3.90 & \pm & 2.07 & 0.83 & 0.3524 & \\
\hline 3-carboxy-4-methyl-5-propyl-2-furanpropanoate & 241.009 & 12.24 & \pm & 5.06 & 1.06 & \pm & 0.43 & 11.60 & $1.66 \mathrm{E}-06$ & $* * *$ \\
\hline oleamide & 282.670 & 0.64 & \pm & 0.94 & 0.91 & \pm & 1.28 & 0.70 & 0.5949 & \\
\hline laurylcarnitine & 344.231 & 3.28 & \pm & 1.49 & 19.95 & \pm & 2.67 & 0.16 & $1.21 \mathrm{E}-12$ & $* * *$ \\
\hline stearoylcarnitine & 428.374 & 21.12 & \pm & 12.52 & 36.96 & \pm & 12.20 & 0.57 & 0.0103 & $*$ \\
\hline palmitoylcarnitine & 400.306 & 4.14 & \pm & 6.85 & 34.33 & \pm & 40.00 & 0.12 & 0.0302 & $*$ \\
\hline 3-dehydrocarnitine & 160.133 & 7.82 & \pm & 2.93 & 18.13 & \pm & 14.88 & 0.43 & 0.0454 & $*$ \\
\hline decanoylcarnitine & 316.775 & 1.12 & \pm & 0.65 & 2.05 & \pm & 1.26 & 0.55 & 0.0536 & \\
\hline carnitine & 162.113 & 577.18 & \pm & 167.08 & 688.78 & \pm & 89.91 & 0.84 & 0.0793 & \\
\hline acetylcarnitine & 204.125 & 404.94 & \pm & 173.55 & 316.09 & \pm & 116.44 & 1.28 & 0.1955 & \\
\hline propionylcarnitine & 218.088 & 0.43 & \pm & 0.53 & 1.15 & \pm & 0.49 & 0.37 & 0.0052 & $*$ \\
\hline isobuthylcarnitine & 233.109 & 4.36 & \pm & 0.70 & 3.83 & \pm & 1.29 & 1.14 & 0.2721 & \\
\hline butyrylcarnitine & 232.158 & 0.44 & \pm & 0.87 & 0.75 & \pm & 1.12 & 0.59 & 0.5031 & \\
\hline isovalerylcarnitine & 246.172 & 3.09 & \pm & 2.38 & 3.74 & \pm & 3.78 & 0.83 & 0.6494 & \\
\hline hexanoylcarnitine & 260.189 & 4.39 & \pm & 2.95 & 3.61 & \pm & 5.42 & 1.22 & 0.6944 & \\
\hline urate & 169.060 & 53.16 & \pm & 14.49 & 82.21 & \pm & 13.79 & 0.65 & $2.27 \mathrm{E}-04$ & $* *$ \\
\hline 1-oleoylglycerophosphocholine & 522.351 & 2749.33 & \pm & 2331.23 & 10948.54 & \pm & 1978.49 & 0.25 & $1.06 \mathrm{E}-07$ & $* * *$ \\
\hline 1-myristoylglycerophosphocholine & 468.305 & 57.61 & \pm & 34.24 & 567.16 & \pm & 196.86 & 0.10 & $2.19 \mathrm{E}-07$ & $* * *$ \\
\hline 1-stearoylglycerophosphocholine & 524.355 & 41.01 & \pm & 27.61 & 181.67 & \pm & 51.83 & 0.23 & $5.29 \mathrm{E}-07$ & $* * *$ \\
\hline 1-palmitoylglycerophosphocholine & 496.327 & 201.50 & \pm & 109.28 & 1601.49 & \pm & 947.66 & 0.13 & $2.03 \mathrm{E}-04$ & $* *$ \\
\hline 1-linoleoylglycerophosphocholine & 520.332 & 5220.85 & \pm & 3503.91 & 11072.58 & \pm & 2457.73 & 0.47 & $4.09 \mathrm{E}-04$ & $* *$ \\
\hline 1-heptadecanoylglycerophosphocholine & 510.358 & 148.35 & \pm & 90.26 & 332.79 & \pm & 116.92 & 0.45 & $9.42 \mathrm{E}-04$ & $* *$ \\
\hline 2-palmitoylglycerophosphocholine & 496.413 & 3.79 & \pm & 3.40 & 53.22 & \pm & 43.48 & 0.07 & 0.0021 & $*$ \\
\hline 2-stearoylglycerophosphocholine & 524.360 & 5.28 & \pm & 3.21 & 21.21 & \pm & 15.56 & 0.25 & 0.0053 & $*$ \\
\hline 1-arachidonoylglycerophosphocholine & 544.323 & 1822.62 & \pm & 1291.58 & 4682.80 & \pm & 2629.36 & 0.39 & 0.0064 & $*$ \\
\hline 2-oleoylglycerophosphocholine & 522.352 & 168.73 & \pm & 84.84 & 833.10 & \pm & 753.91 & 0.20 & 0.0126 & $*$ \\
\hline 1-eicosatrienoylglycerophosphocholine & 546.356 & 79.42 & \pm & 56.88 & 188.46 & \pm & 132.88 & 0.42 & 0.0283 & $*$ \\
\hline 1-palmitoleoylglycerophosphocholine & 496.802 & 4.16 & \pm & 3.49 & 7.26 & \pm & 2.26 & 0.57 & 0.0296 & $*$ \\
\hline glycerophosphorylcholine & 258.821 & 2.25 & \pm & 0.77 & 1.65 & \pm & 0.43 & 1.36 & 0.0469 & $*$ \\
\hline glycerol 3-phosphate (G3P) & 173.052 & 57.38 & \pm & 4.83 & 52.48 & \pm & 10.70 & 1.09 & 0.2038 & \\
\hline glycerol & 93.058 & 1.31 & \pm & 0.63 & 1.17 & \pm & 0.78 & 1.12 & 0.6531 & \\
\hline 1-stearoylglycerol & 359.335 & 34.43 & \pm & 21.00 & 57.37 & \pm & 17.22 & 0.60 & 0.0156 & $*$ \\
\hline hyodeoxycholate & 393.211 & 23.99 & \pm & 5.83 & 111.87 & \pm & 13.31 & 0.21 & $2.08 \mathrm{E}-13$ & $* * *$ \\
\hline cholate & 409.177 & 0.00 & \pm & 0.00 & 1.62 & \pm & 0.45 & 0.00 & $1.11 \mathrm{E}-09$ & $* * *$ \\
\hline taurochenodeoxycholate & 500.278 & 2.07 & \pm & 2.50 & 19.29 & \pm & 9.44 & 0.11 & $2.71 \mathrm{E}-05$ & $* *$ \\
\hline glycochenodeoxycholate & 450.323 & 2.81 & \pm & 3.48 & 53.31 & \pm & 32.00 & 0.05 & $1.01 \mathrm{E}-04$ & $* *$ \\
\hline ursodeoxycholate & 393.298 & 5.25 & \pm & 2.49 & 6.36 & \pm & 1.85 & 0.83 & 0.2727 & \\
\hline androsterone sulfate & 371.230 & 4.66 & \pm & 2.01 & 31.02 & \pm & 3.13 & 0.15 & $1.36 \mathrm{E}-14$ & $* * *$ \\
\hline cortisol & 365.204 & 28.67 & \pm & 8.44 & 6.37 & \pm & 6.78 & 4.50 & $4.00 \mathrm{E}-06$ & $* * *$ \\
\hline cholesterol & 387.362 & 0.69 & \pm & 0.31 & 2.44 & \pm & 2.21 & 0.28 & 0.0231 & $*$ \\
\hline dehydroisoandrosterone sulfate & 369.292 & 63.60 & \pm & 87.00 & 254.90 & \pm & 243.68 & 0.25 & 0.0311 & $*$ \\
\hline cortisone & 363.304 & 0.64 & \pm & 0.65 & 3.09 & \pm & 3.31 & 0.21 & 0.0336 & $*$ \\
\hline Energy & & & & & & & & & & \\
\hline citrate & 119.091 & 7.55 & \pm & 4.02 & 12.60 & \pm & 6.12 & 0.60 & $3.47 \mathrm{E}-06$ & $* *$ \\
\hline alpha-ketoglutarate & 147.050 & 74.72 & \pm & 9.45 & 68.94 & \pm & 4.16 & 1.08 & 0.0935 & \\
\hline acetylphosphate & 141.090 & 9.47 & \pm & 0.85 & 10.68 & & 0.90 & 0.89 & 0.0062 & $*$ \\
\hline Nucleotide Metabolism & & & & & & & & & & \\
\hline 1-methyladenosine & 282.205 & 36.95 & \pm & 8.04 & 18.78 & \pm & 5.11 & 1.97 & $1.05 \mathrm{E}-05$ & $* * *$ \\
\hline adenosine monophosphate & 348.067 & 11.03 & \pm & 8.74 & 1.91 & \pm & 2.90 & 5.78 & 0.0058 & $*$ \\
\hline guanosine & 284.274 & 28.86 & \pm & 13.56 & 11.40 & \pm & 13.50 & 2.53 & 0.0098 & $*$ \\
\hline deoxyguanosine & 268.237 & 21.03 & \pm & 3.33 & 17.47 & \pm & 2.45 & 1.20 & 0.0140 & $*$ \\
\hline thymidine monophosphate & 323.253 & 15.34 & \pm & 2.24 & 18.87 & \pm & 12.76 & 0.81 & 0.3997 & \\
\hline uridine monophosphate & 325.237 & 12.39 & \pm & 4.47 & 9.34 & \pm & 1.83 & 1.33 & 0.0608 & \\
\hline deoxy uridine monophosphate & 309.201 & 0.87 & \pm & 0.59 & 2.59 & \pm & 8.20 & 0.34 & 0.5171 & \\
\hline pseudouridine & 245.083 & 4.39 & \pm & 3.05 & 0.59 & \pm & 0.34 & 7.45 & 0.0010 & $*$ \\
\hline
\end{tabular}


a) arginine

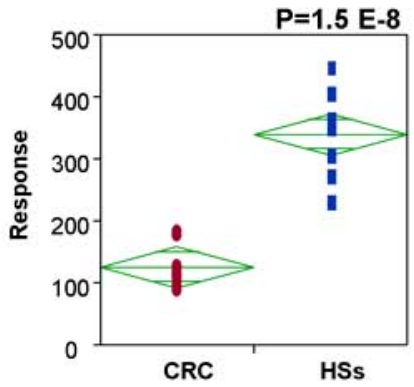

e) $m / z 224.868$ rt 2.06

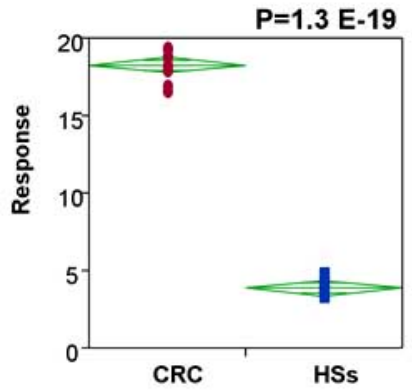

b) histidine

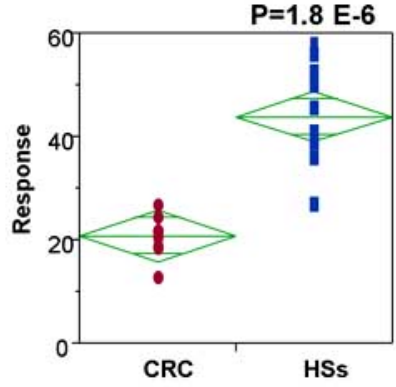

f) $\mathrm{m} / \mathrm{z} 96.922$ rt 2.06

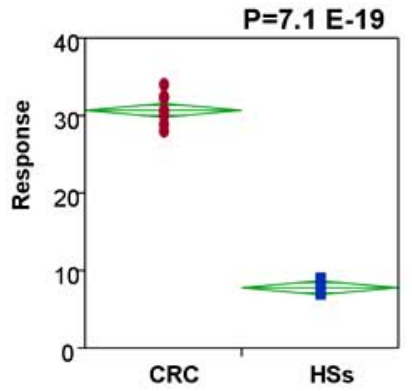

\section{c) threonine}

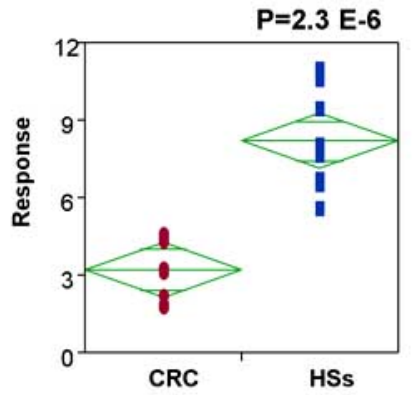

g) $m / z 416.809$ rt 2.08

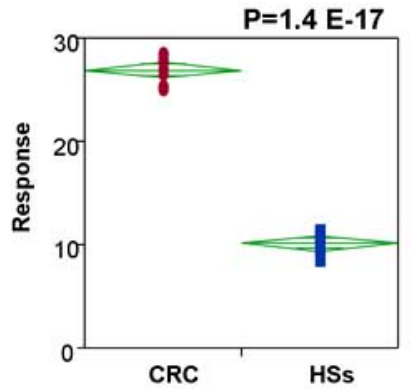

d) p-cresol sulfate

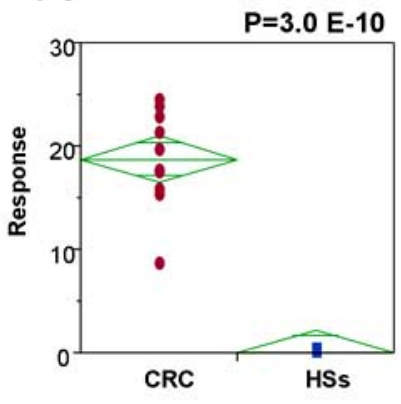

h) $m / z 943.221$ rt 2.39

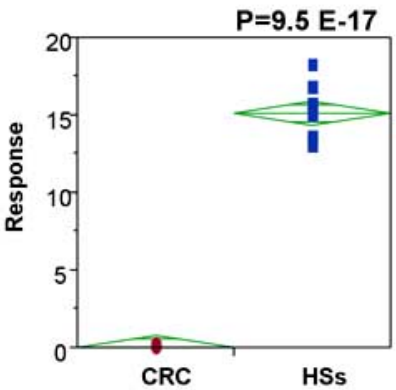

Fig. (2). Scatter plot of expressed tendency and regularity of LMCs (significant difference) between colorectal cancer (CRC) patients group and healthy subjects (HSs) group. CRC patients showed decreases in arginine, histidine, threonine, and unidentified LMC $(\mathrm{m} / z ; 943.221)$ levels and increases in p-cresol sulfate, and unidentified LMC $(m / z ; 224.868,96.922,416.809)$ levels compared with HSs.

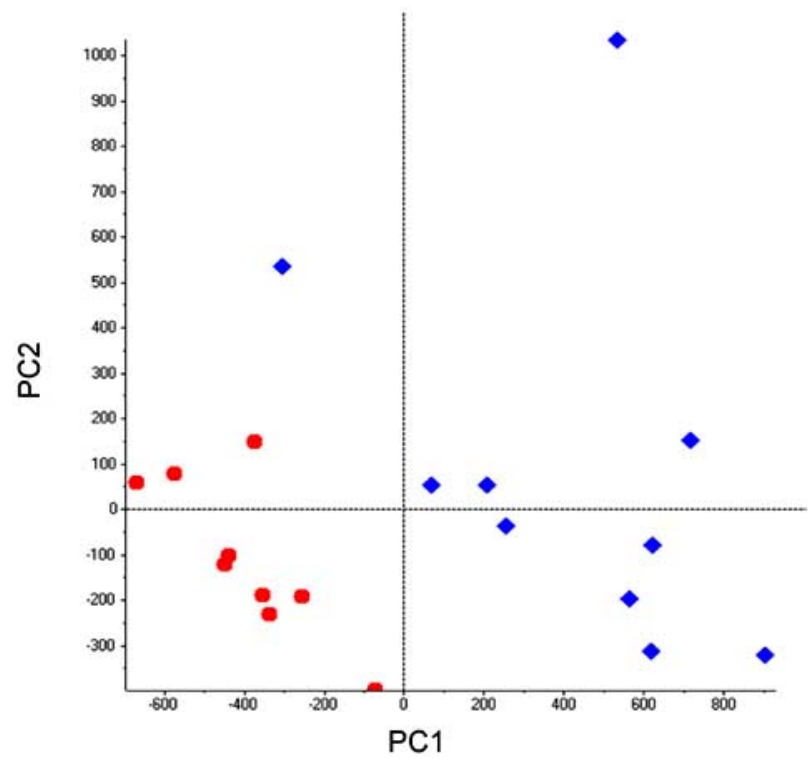

Fig. (3). Score plot of unsupervised principal component analysis of CRC patients (red circles) and healthy subjects (blue diamonds) using metabolomic data. Total analysis of PCA indicated that whole metabolic pattern differed between CRC patients and healthy subjects.

of the hypoxic conditions of CRC tumor tissue compared with corresponding normal mucosa [7]. Denkert et al. found that intermediates of the TCA cycle and lipids were down- regulated in cancerous tissue, whereas urea cycle metabolites, purines, pyrimidines, and amino acids were increased compared to normal mucosa [5]. The results of the present study indicate that decreased CRC blood plasma levels of many free amino acids are related to tumoral changes in amino acid concentrations. These amino acid decreases could be due to their removal from the circulation by cancer cells for direct use in tumor growth or their rapid metabolization for tumoral energy production. The latter may be more plausible as significant increases in CRC blood plasma free fatty acids may also indicate the higher energy demands of the tumor. Moreover, increased CRC blood plasma levels of nucleotides such as 1-methyl adenosine and adenosine 5'-monophosphate could represent a high DNA/RNA turnover in tumoral tissues. Together, these findings suggest that tumoral specific metabolite changes are reflected in alterations of blood plasma metabolites, the analysis of which could form a CRC tumoral metabolic profile.

Several fibrinogen-derived peptides were significantly (weakly and moderately) elevated in the CRC patients group. These results appear to agree with raised fibrinogen levels found in various cancers such as breast cancer, lung cancer, and gastric cancer [22-24]. It has been suggested that increased 2-aminobutyrate levels in blood plasma reflect oxidative stress in cancer patients. Moreover, stress-related metabolites such as cortisol and glycerophosphorylchorine were increased in the CRC patients group. Interestingly, the uremic toxins p-cresol sulfate and 3-calboxy-4- methyl-5- 


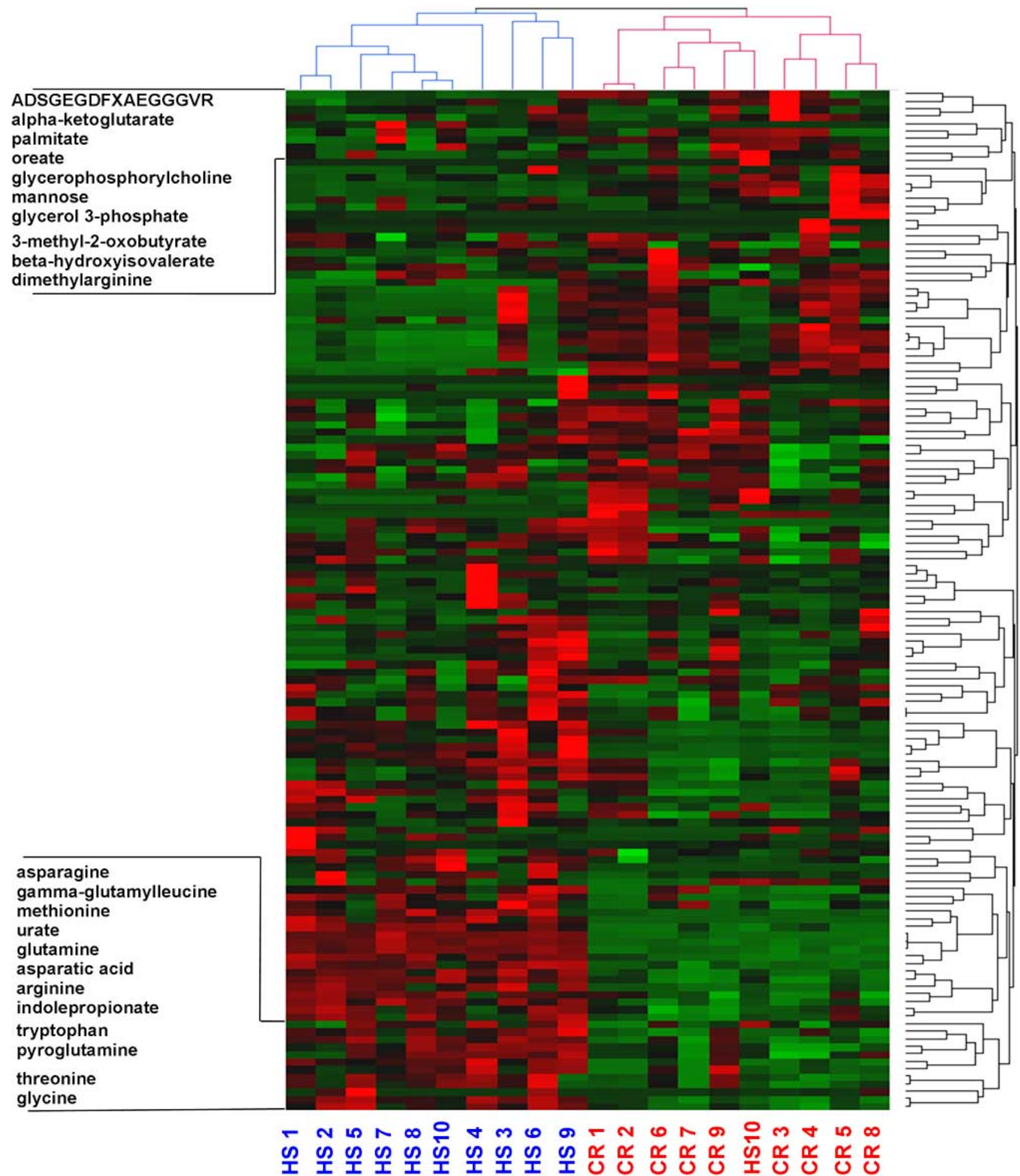

Fig. (4). Unsupervised hierarchical clustering of CRC patients and healthy subjects with respect to 130 metabolites. Heatmap visualizes metabolite abundance in each sample ranging from high (red) to average (black) to low (green).

propyl-2-furanpropanate were also significantly elevated in CRC blood plasma, which could result from inefficient filtration as a consequence of renal stress. In particular, pcresol sulfate was detected only in the CRC patients group.
These uremic toxins are also known to bind albumin, suggesting that a change in the blood plasma metabolite profiles of CRC patients could dislodge them from albumin. 
Metabolomics can be described as a global approach, with a main aim to compare large sample sets based on changes in the concentration profile of a multitude of LMCs known as metabolites $[16,17,25]$. The technique also relies on multiple sample comparisons, systematic detection between samples, prediction of independent sample sets, metabolites pattern, and identification of key metabolites. In general, smaller peaks are more sensitive to interference, so are distorted and result in unstable values of peak areas [26]. To ensure stability, sample preparation before LC-TOF/MS analysis should be performed carefully. In the present metabolomic study, all samples were prepared using the same protocol to ensure repeatability and technical replicates were performed three times. Almost all corresponding peaks were presented at similar retention times (less than $0.1 \mathrm{~min}$ ), which was of benefit in matching and extracting the coeluting peaks. Because of the intensity from MS analysis containing variations from each injection, it is important to normalize the obtained data for the comparison of metabolomic data. In this study, obtained data were normalized using total intensity of each injection. Compared with the normalization methods used median peak intensity, known metabolite intensity as an internal standard, and the total peak intensity, using total intensity of all peaks were most good repeatability.

Combining blood plasma metabolites aggregation data with PCA and hierarchical clustering enables the separation of CRC patients from healthy subjects. Both multivariate analyses characterize CRC patients using detected 4,190 peaks for PCA and identified 130 LMCs for hierarchical clustering. Amino acids, such as glycine, threonine, tryptophan, arginine and asparatic acid, were clustered lower half of figure. Plasma amino acids levels may important for the diagnose CRC patients from healthy subjects. Although the metabolomic approach of the present study is a useful tool for characterizing CRC patients, it is not without its limitations. First, the metabolites identified in our study were only a subset of the total LMCs, so providing only a limited metabolic profile of CRC. Second, the relationship between blood plasma metabolites and CRC tissues from the same patient was not investigated. Third, blood plasma samples were obtained from CRC patients and healthy subjects with non-regulated dietary conditions and environmental factors. Future metabolomic studies should take these factors into account and evaluate a larger number of plasma samples to validate our current results.

This study indicates that the LC-TOF/MS technique is a valuable tool for metabolomic study, an approach that will provide theoretical evidence for monitoring tumoral metabolite changes using blood plasma to characterize CRC patients. Metabolic profiling studies of the direct relationship between tumoral changes and blood plasma are not available yet, but we believe they will become common practice in monitoring the tumor situation. Current information about LMC changes in tissue and/or blood plasma of CRC is only limited, so it is important to utilize technical advances and data driven research to explore metabolic changes, to develop methods for early cancer detection, and to obtain a fuller understanding of cancer mechanisms with the aim of increasing patient survival rates.

\section{CONCLUSIONS}

This study employed a metabolomic analysis using LCTOF/MC of blood plasma from CRC patients and normal subjects. Multivariate analysis revealed different and distinctive LMC profiles for both groups. Some identified LMCs in blood plasma agree with previously reported tumoral metabolite changes. The study confirms the metabolomic technique as a practical method for understanding the disease character of CRC.

\section{ABBREVIATIONS}

$\begin{array}{ll}\text { CA19-9 } & \text { Carbohydrate antigen 19-9 } \\ \text { CEA } & =\text { Carcinoembryonic antigen } \\ \text { LC-TOF/MS }= & \text { Liquid chromatography-time of flight } / \text { mass } \\ & \text { spectrometry } \\ \text { LMCs } & =\text { Low molecular weight compounds }\end{array}$

\section{REFERENCES}

[1] Parkin, D.M.; Bray, F.; Ferlay, J.; Pisani, P. Global cancer statistics 2002. CA Cancer J. Clin., 2005, 55(2), 74-108.

[2] Ward, D.G.; Suggett, N.; Cheng, Y.; Wei, W.; Johnson, H.; Billingham, L.J.; Ismail, T.; Wakelam, M.J.; Johnson, P.J.; Martin, A. Identification of serum biomarkers for colon cancer by proteomic analysis. Br. J. Cancer, 2006, 94(12), 1898-1905.

[3] de Noo, M.E.; Tollenaar, R.A.; Deelder, A.M.; Bouwman, L.H. Current status and prospects of clinical proteomics studies on detection of colorectal cancer: hopes and fears. World J. Gastroenterol., 2006, 12(41), 6594-6601.

[4] Mazzanti, R.; Solazzo, M.; Fantappié O.; Elfering S.; Pantaleo P.; Bechi P.; Cianchi F.; Ettl A.; Giulivi C. Differential expression proteomics of human colon cancer. Am. J. Physiol. Gastrointest Liver Physiol., 2006, 290(6), G1329-1338.

[5] Denkert, C.; Budczies, J.; Weichert, W.; Wohlgemuth, G.; Scholz, M.; Kind, T.; Niesporek, S.; Noske, A.; Buckendahl, A.; Dietel, M.; Fiehn, O. Metabolite profiling of human colon carcinoma-deregulation of TCA cycle and amino acid turnover. Mol. Cancer, 2008, 7, 72 .

[6] Chan, E.C.; Koh, P.K.; Mal, M.; Cheah, P.Y.; Eu, K.W.; Backshall, A.; Cavill, R.; Nicholson, J.K.; Keun, H.C. Metabolic profiling of human colorectal cancer using high-resolution magic angle spinning nuclear magnetic resonance (HR-MAS NMR) spectroscopy and gas chromatography mass spectrometry (GC/MS). J. Proteome Res., 2009, 8(1), 352-361.

[7] Hirayama, A; Kami, K; Sugimoto, M; Sugawara, M; Toki, N; Onozuka, H; Kinoshita, T; Saito, N; Ochiai, A; Tomita, M; Esumi, $\mathrm{H}$; Soga, T. Quantitative metabolome profiling of colon and stomach cancer microenvironment by capillary electrophoresis time-of-flight mass spectrometry. Cancer Res., 2009, 69(11), 49184925.

[8] Mal, M.; Koh, P.K.; Cheah, P.Y.; Chan, E.C. Development and validation of a gas chromatography/mass spectrometry method for the metabolic profiling of human colon tissue. Rapid Commun. Mass Spectrom., 2009, 23(4), 487-494.

[9] Qiu, Y.; Cai, G.; Su, M.; Chen, T.; Zheng, X.; Xu, Y.; Ni, Y.; Zhao, A.; Xu, L.X.; Cai, S.; Jia, W. Serum metabolite profiling of human colorectal cancer using GC-TOFMS and UPLC-QTOFMS. J. Proteome. Res., 2009, 8(10), 4844-4850.

[10] A, J.; Trygg, J; Gullberg, J.; Johansson, A.I.; Jonsson, P.; Antti, H.; Marklund, S.L.; Moritz, T. Extraction and GC/MS analysis of the human blood plasma metabolome. Anal. Chem., 2005, 77(24): 8086-8094.

[11] Montoliu, I.; Martin, F.P.; Collino, S.; Rezzi, S.; Kochhar, S. Multivariate modeling strategy for intercompartmental analysis of tissue and plasma 1H NMR spectrotypes. J. Proteome Res., 2009, 8(5), 2397-2406.

[12] Soga, T.; Baran, R.; Suematsu, M.; Ueno, Y.; Ikeda, S.; Sakurakawa, T.; Kakazu, Y.; Ishikawa, T.; Robert, M.; Nishioka, T.; Tomita, M. Differential metabolomics reveals ophthalmic acid as an oxidative stress biomarker indicating hepatic glutathione consumption. J. Biol. Chem., 2006, 281(24), 16768-16776. 
[13] Seierstad, T.; Røe, K.; Sitter, B.; Halgunset, J.; Flatmark, K.; Ree, A.H.; Olsen, D.R.; Gribbestad, I.S.; Bathen, T.F. Principal component analysis for the comparison of metabolic profiles from human rectal cancer biopsies and colorectal xenografts using highresolution magic angle spinning $1 \mathrm{H}$ magnetic resonance spectroscopy. Mol. Cancer, 2008, 7, 33 .

[14] Pasikanti, K.K.; Ho, P.C.; Chan, E.C. Gas chromatography/mass spectrometry in metabolic profiling of biological fluids. $J$. Chromatogr. B Analyt Technol. Biomed. Life Sci., 2008, 871(2), 202-211.

[15] Ma, Y.; Liu, W.; Peng, J.; Huang, L.; Zhang, P.; Zhao, X.; Cheng, Y.; Qin, H. A pilot study of gas chromatograph/mass spectrometrybased serum metabolic profiling of colorectal cancer after operation. Mol. Biol. Rep., 2009, doi 10.1007/s11033-009-9524-4.

[16] Wilson, I.D.; Plumb, R.; Granger, J.; Major, H.; Williams, R.; Lenz, E.M. HPLC-MS-based methods for the study of metabonomics. J. Chromatogr. B Analyt. Technol. Biomed. Life Sci., 2005, 817(1), 67-76.

[17] Claudino, W.M.; Quattrone, A.; Biganzoli, L.; Pestrin, M.; Bertini, I.; Di Leo, A. Metabolomics: available results, current research projects in breast cancer, and future applications. J. Clin. Oncol., 2007, 25(19), 2840-2846.

[18] Xue, R.; Lin, Z.; Deng, C.; Dong, L.; Liu, T.; Wang, J.; Shen, X. A serum metabolomic investigation on hepatocellular carcinoma patients by chemical derivatization followed by gas chromatography/mass spectrometry. Rapid Commun. Mass Spectrom., 2008, 22(19), 3061-3068.

[19] Woo, H.M.; Kim, K.M.; Choi, M.H.; Jung, B.H.; Lee, J.; Kong, G.; Nam, S.J.; Kim, S.; Bai, S.W.; Chung, B.C. Mass spectrometry based metabolomic approaches in urinary biomarker study of women's cancers. Clin. Chim. Acta., 2009, 400(1-2), 63-69.
[20] Sreekumar, A.; Poisson, L.M.; Rajendiran, T.M.; Khan, AP.; Cao, Q.; Yu, J.; Laxman, B.; Mehra, R.; Lonigro, R.J.; Li, Y.; Nyati, M.K.; Ahsan, A.; Kalyana-Sundaram, S.; Han, B.; Cao, X.; Byun, J.; Omenn, G.S.; Ghosh, D.; Pennathur, S.; Alexander, D.C.; Berger, A.; Shuster, J.R.; Wei, J.T.; Varambally, S.; Beecher, C.; Chinnaiyan, A.M. Metabolomic profiles delineate potential role for sarcosine in prostate cancer progression. Nature, 2009, 457(7231), 910-914.

[21] Buscher, J.M.; Czernik, D.; Ewald, J.C.; Sauer, U.; Zamboni, N. Cross-platform comparison of methods for quantitative metabolomics of primary metabolism. Anal. Chem., 2009, 81(6), 2135-2143.

[22] Dirix, L.Y.; Salgado, R.; Weytjens, R.; Colpaert, C.; Benoy, I.; Huget, P.; van Dam, P.; Prové, A.; Lemmens, J.; Vermeulen, P. Plasma fibrin D-dimer levels correlate with tumour volume, progression rate and survival in patients with metastatic breast cancer. Br. J. Cancer, 2002, 86(3), 389-395.

[23] Taguchi, O.; Gabazza, E.C.; Yasui, H.; Kobayashi, T.; Yoshida, M.; Kobayashi, H. Prognostic significance of plasma D-dimer levels in patients with lung cancer. Thorax, 1997, 52(6), 563-565.

[24] Yamashita, H.; Kitayama, J.; Nagawa, H. Hyperfibrinogenemia is a useful predictor for lymphatic metastasis in human gastric cancer. Jpn. J. Clin. Oncol., 2005, 35(10), 595-600.

[25] Nicholson, J.K.; Lindon, J.C.; Holmes, E. Metabonomics: understanding the metabolic responses of living systems to pathophysiological stimuli via multivariate statistical analysis of biological NMR spectroscopic data. Xenobiotica, 1999, 29(11), 1181-1189.

[26] Yu, K.; Sheng, G.; Sheng, J.; Chen, Y.; Xu, W.; Liu, X.; Cao, H.; $\mathrm{Qu}, \mathrm{H}$.; Cheng, Y.; Li, L. A metabonomic investigation on the biochemical perturbation in liver failure patients caused by hepatitis B virus. J. Proteome Res., 2007, 6(7), 2413-2419.

(C) Fukui and Itoh; Licensee Bentham Open.

This is an open access article licensed under the terms of the Creative Commons Attribution Non-Commercial License (http://creativecommons.org/licenses/by-nc/ 3.0/) which permits unrestricted, non-commercial use, distribution and reproduction in any medium, provided the work is properly cited. 